\title{
Quasiparticle states driven by a scattering on the preformed electron pairs**
}

\author{
T. Domański \\ Institute of Physics, M. Curie-Skłodowska University, 20-031 Lublin, Poland \\ Received November 13, 2015, in final form November 27, 2015 \\ We analyze evolution of the single particle excitation spectrum of the underdoped cuprate superconductors \\ near the anti-nodal region, considering temperatures below and and above the phase transition. We inspect the \\ phenomenological self-energy that reproduces the angle-resolved-photoemission-spectroscopy (ARPES) data \\ and we show that above the critical temperature, such procedure implies a transfer of the spectral weight \\ from the Bogoliubov-type quasiparticles towards the in-gap damped states. We also discuss some possible \\ microscopic arguments explaining this process.
}

Key words: superconducting fluctutations, Bogoliubov quasiparticles, pseudogap

PACS: $74.20 .-z, 74.20 . \mathrm{Mn}, 74.40 .+k$

\section{Introduction}

Superconductivity (i.e., dissipationless motion of the charge carriers) is observed at sufficiently low temperatures, when electrons from the vicinity of the Fermi surface are bound in the pairs and respond collectively (rather than individually) to any external perturbation such as electromagnetic field, pressure, temperature gradient, etc. Depending on specific materials, the pairing mechanism can be driven by phonons (in classical superconductors), magnons (in heavy fermion compounds) or by the antiferromagnetic exchange interactions originating from the Coulomb repulsion (in cuprate oxides). In most cases, the electron pairs are formed at the critical value $T_{\mathrm{c}}$, marking the onset of superconductivity. There are, however, numerous exceptions to this rule. For instance, in the cuprate superconductors [1] or in the ultracold fermionic gasses [2], such pairs pre-exist well above $T_{\mathrm{c}}$. To some extent, their presence causes the properties reminiscent of the superconducting state.

Early evidence for the preformed pairs existing above $T_{\mathrm{c}}$ has been indicated in the muon scattering experiments [3]. Later on, their existence was supported by the ultrafast (tera-Hertz) optical spectroscopy [4, 5] and the large Nernst effect [6, 7]. Spectroscopic signatures of the preformed pairs have been also detected directly in the ARPES measurements on yttrium [8] and lanthanum [9] cuprate oxides, revealing the Bogoliubov-type quasiparticle dispersion above $T_{\mathrm{c}}$. Furthermore, the STM imaging provided clear fingerprints of such dispersive Bogoliubov quasiparticles (by the unique octet patterns) being unchanged from temperatures deep in the superconducting region up to $1.5 T_{\mathrm{c}}$ [10]. Superconducting fluctuations above $T_{\mathrm{c}}$ have been also reported by the Josephson-like tunneling [11] and the proximity effect induced in the nanosize metallic slabs deposited on $\mathrm{La}_{2-x} \mathrm{Sr}_{x} \mathrm{CuO}_{4}$ [12]. More recently, the residual Meissner effect has been experimentally observed above the transition temperature $T_{\mathrm{c}}$ by the torque magnetometry [13] and other measurements [14,15]. Additional evidence for the superconducting-like behaviour above $T_{\mathrm{c}}$ has been seen in the high-resolution ARPES measurements [16], the superfulid fraction observed in the $c$-axis optical measurements $\operatorname{Re}\left\{\sigma_{\mathrm{c}}(\omega)\right\}$ [17], the Josephson spectroscopy for YBaCuO-LaSrCuO-YBaCuO junction using LaSrCuO in the pseudogap state well above $T_{\mathrm{c}}$ [18], optical conductance in the pseudogap state of $\mathrm{YBaCuO}$ superconductor [19] and the photo-enhanced antinodal conductivity in pseudogap state of the high $T_{\mathrm{c}}$ superconductors [20].

\footnotetext{
${ }^{*}$ This work is dedicated to professor Stefan Sokołowski on the occasion of his 65-th birthday.
} 
Preformed pairs are correlated above $T_{\mathrm{c}}$ only on some short temporal $\tau_{\phi}$ and spatial $l_{\phi}$ scales [2123]. For this reason, the superconducting fluctuations are manifested in very peculiar way [24]. Their influence on the single particle spectrum is manifested by: a) two Bogoliubov-type branches and b) additional in-gap states that are over-damped (have a short life-time). Temperature has a strong effect on the transfer of the spectral weight between these entities. In the underdoped cuprate superconductors, such a transfer is responsible for filling-in the energy gap [16, 25], instead of closing it (as in the classical superconductors). Some early results concerning superconducting fluctuations have been known for a long time [26, 27], but they attracted much more interest in the context of cuprate superconductors [26-36] and ultracold fermion superfluids [37, 38].

In this work, we study qualitative changeover of the single particle electronic spectrum of the underdoped cuprate oxides for temperatures varying from below $T_{\mathrm{C}}$ (in the superconducting state) to above $T_{\mathrm{c}}$, where the preformed pairs are not long-range coherent. In the superconducting state, the usual Bogoliubov-type quasiparticles are driven by the Bose-Einstein condensate of the (zero-momentum) Cooper pairs. We find that above $T_{\mathrm{c}}$, the Bogoliubov quasiparticles are still preserved, but the scattering processes driven by the finite momentum pairs contribute the in-gap states whose life-time substantially increases with increasing temperature. We discuss this process on the phenomenological as well as microscopic arguments. Roughly speaking, a feedback of the electron pairs on the unpaired electrons resembles the long-range translational and orientational order that develops between the amphiphilic particles in presence of the ions at solid state surfaces studied by S. Sokołowski with coworkers [39].

\section{Microscopic formulation of the problem}

To account for the coherent/incoherent pairing we consider the Hamiltonian

$$
\hat{H}=\sum_{\mathbf{k}, \sigma}\left(\varepsilon_{\mathbf{k}}-\mu\right) \hat{c}_{\mathbf{k} \sigma}^{\dagger} \hat{c}_{\mathbf{k} \sigma}+\frac{1}{N} \sum_{\mathbf{k}, \mathbf{k}^{\prime}, \mathbf{q}} V_{\mathbf{k}, \mathbf{k}^{\prime}}(\mathbf{q}) \hat{c}_{\mathbf{k}^{\prime} \uparrow}^{\dagger} \hat{c}_{\mathbf{q}-\mathbf{k}^{\prime} \downarrow}^{\dagger} \hat{c}_{\mathbf{q}-\mathbf{k} \downarrow} \hat{c}_{\mathbf{k} \uparrow}
$$

describing the mobile electrons of kinetic energy $\varepsilon_{\mathbf{k}}$ (where $\mu$ is the chemical potential) interacting via the two-body potential $V_{\mathbf{k}, \mathbf{k}^{\prime}}(\mathbf{q})$. We assume a separable form $V_{\mathbf{k}, \mathbf{k}^{\prime}}=-g \eta_{\mathbf{k}} \eta_{\mathbf{k}^{\prime}}$ of this pairing potential (with $g>0)$. In the nearly two-dimensional cuprate superconductors with the prefactor $\eta_{\mathbf{k}}=\frac{1}{2}\left[\cos \left(a k_{x}\right)+\cos \left(a k_{y}\right)\right]$ (where $a$ is the unit length in $\mathrm{CuO}_{2}$ planar structure), such pairing potential induces the $d$-wave symmetry order parameter [40, 41].

The Hamiltonian (2.1) can be recast in a more compact form, by introducing the pair operators

$$
\hat{b}_{\mathbf{q}}=\frac{1}{\sqrt{N}} \sum_{\mathbf{k}} \eta_{\mathbf{k}} \hat{c}_{\mathbf{q}-\mathbf{k} \downarrow} \hat{c}_{\mathbf{k} \uparrow}
$$

and $\hat{b}_{\mathbf{q}}^{\dagger}=\left(\hat{b}_{\mathbf{q}}\right)^{\dagger}$, when the two-body interactions simplify to

$$
\frac{1}{N} \sum_{\mathbf{k}, \mathbf{k}^{\prime}, \mathbf{q}} V_{\mathbf{k}, \mathbf{k}^{\prime}}(\mathbf{q}) \hat{c}_{\mathbf{k} \uparrow}^{\dagger} \hat{c}_{\mathbf{q}-\mathbf{k} \downarrow}^{\dagger} \hat{c}_{\mathbf{q}-\mathbf{k}^{\prime} \downarrow} \hat{c}_{\mathbf{k}^{\prime} \uparrow}=-\sum_{\mathbf{q}} g \hat{b}_{\mathbf{q}}^{\dagger} \hat{b}_{\mathbf{q}}
$$

Using the Heisenberg equation of motion $(\hbar=1)$

$$
\mathrm{i} \frac{\mathrm{d}}{\mathrm{d} t} \hat{c}_{\mathbf{k} \uparrow}=\left(\varepsilon_{\mathbf{k}}-\mu\right) \hat{c}_{\mathbf{k} \uparrow}-g \eta_{\mathbf{k}} \frac{1}{\sqrt{N}} \sum_{\mathbf{q}} \hat{c}_{\mathbf{q}-\mathbf{k} \downarrow}^{\dagger} \hat{b}_{\mathbf{q}}
$$

we immediately notice that the single-particle properties of this model (2.1):

a) are characterized by the mixed particle and hole degrees of freedom (because the annihilation operators $\hat{c}_{\mathbf{k} \uparrow}$ couple to the creation operators $\hat{c}_{\mathbf{q}-\mathbf{k} \downarrow}^{\dagger}$ ),

b) depend on the pairing field $\hat{b}_{\mathbf{q}}$ (appearing in the equation of motion $\frac{\mathrm{d}}{\mathrm{d} t} \hat{c}_{\mathbf{k} \uparrow}$ ).

Both these features manifest themselves in the superconducting state, when there exists the Bose-Einstein (BE) condensate $\left\langle\hat{b}_{\mathbf{q}=\mathbf{0}}\right\rangle \neq 0$ of the Cooper pairs. They also survive in the normal state, as long as the preformed (finite-momentum) pairs are present below the same characteristic temperature $T^{*}$ marking an onset of the electron pairing. In the next section we explore their role in the superconducting state $\left(T \leqslant T_{\mathrm{C}}\right)$ and in the pseudogap region $\left(T_{\mathrm{c}}<T<T^{*}\right)$. 


\section{Pairs as the scattering centers}

The Heisenberg equation of motion (2.4) indicates that the electronic states are affected by the pairing field $\hat{b}_{\mathbf{q}}$. Let us consider the generic consequences of such Andreev-type scattering, separately considering: the BE condensed $\mathbf{q}=\mathbf{0}$ and the finite-momentum $\mathbf{q} \neq \mathbf{0}$ pairs.

\subsection{The effect of the Bose-Einstein condensed pairs}

We start by considering the usual BCS approach, when only the zero momentum pairs are taken into account. This situation has a particularly clear interpretation within the path integral formalism, treating the pairing field via the Hubbard-Stratonovich transformation and determining it from the minimization of action (the saddle point solution). The same result can be obtained using the equation of motion (2.4), focusing on the effect of $\mathbf{q}=\mathbf{0}$ pairs

$$
\begin{aligned}
\mathrm{i} \frac{\mathrm{d}}{\mathrm{d} t} \hat{c}_{\mathbf{k} \uparrow} \simeq\left(\varepsilon_{\mathbf{k}}-\mu\right) \hat{c}_{\mathbf{k} \uparrow}-g \eta_{\mathbf{k}} \hat{c}_{-\mathbf{k} \downarrow}^{\dagger} \frac{\hat{b}_{\mathbf{0}}}{\sqrt{N}}, \\
\mathrm{i} \frac{\mathrm{d}}{\mathrm{d} t} \hat{c}_{\mathbf{k} \downarrow}^{\dagger} \simeq-\left(\varepsilon_{\mathbf{k}}-\mu\right) \hat{c}_{\mathbf{k} \downarrow}^{\dagger}-g \eta_{\mathbf{k}} \frac{\hat{b}_{\mathbf{0}}^{\dagger}}{\sqrt{N}} \hat{c}_{\mathbf{k} \uparrow} .
\end{aligned}
$$

Macroscopic occupancy of the $\mathbf{q}=\mathbf{0}$ state implies that the bosonic operators $\hat{b}_{\mathbf{0}}^{(\dagger)}$ can be treated as complex numbers $b_{0}^{(*)}$. By introducing the order parameter

$$
\Delta_{\mathbf{k}}=g \eta_{\mathbf{k}} \frac{\left\langle\hat{b}_{\mathbf{0}}\right\rangle}{\sqrt{N}}=g \eta_{\mathbf{k}} \frac{1}{N} \sum_{\mathbf{k}^{\prime}} \eta_{\mathbf{k}^{\prime}}\left\langle\hat{c}_{-\mathbf{k}^{\prime} \downarrow} \hat{c}_{\mathbf{k}^{\prime} \uparrow}\right\rangle
$$

the equations (3.1), 3.2) can be solved exactly using the standard Bogoliubov-Valatin transformation. In such BCS approach, the classical superconductivity has close analogy with the superfluidity of weakly interacting bosons, whose collective sound-like mode originates from the interaction between the finitemomentum bosons and the BE condensate.

In the present context, the zero-momentum Copper pairs substantially affect the single particle excitation spectrum (and the two-body correlations as well). The single-particle Green's function $\boldsymbol{G}(\mathbf{k}, \tau)=$ $-\hat{T}_{\tau}\left\langle\hat{c}_{\mathbf{k} \uparrow}(\tau) \hat{c}_{\mathbf{k} \uparrow}^{\dagger}\right\rangle$, where $\hat{T}_{\tau}$ is the time ordering operator, obeys the Dyson equation

$$
[\boldsymbol{G}(\mathbf{k}, \omega)]^{-1}=\omega-\varepsilon_{\mathbf{k}}+\mu-\Sigma(\mathbf{k}, \omega),
$$

with the BCS self-energy

$$
\Sigma(\mathbf{k}, \omega)=\frac{\left|\Delta_{\mathbf{k}}\right|^{2}}{\omega+\left(\varepsilon_{\mathbf{k}}-\mu\right)}
$$

The self-energy (3.5), accounting for the Andreev-type scattering of the k-momentum electrons on the Cooper pairs, can be alternatively obtained from the bubble diagram. The related spectral function $A(\mathbf{k}, \omega)=-\pi^{-1} \operatorname{Im} \boldsymbol{G}\left(\mathbf{k}, \omega+\mathrm{i}^{+}\right)$is thus characterized by the two-pole structure

$$
A(\mathbf{k}, \omega)=u_{\mathbf{k}}^{2} \delta\left(\omega-E_{\mathbf{k}}\right)+v_{\mathbf{k}}^{2} \delta\left(\omega+E_{\mathbf{k}}\right)
$$

with the Bogoliubov-type quasiparticle energies $E_{\mathbf{k}}= \pm \sqrt{\left(\varepsilon_{\mathbf{k}}-\mu\right)^{2}+\Delta_{\mathbf{k}}^{2}}$ and the spectral weights $u_{\mathbf{k}}^{2}=$ $\frac{1}{2}\left[1+\left(\varepsilon_{\mathbf{k}}-\mu\right) / E_{\mathbf{k}}\right]$ and $v_{\mathbf{k}}^{2}=1-u_{\mathbf{k}}^{2}$. Let us remark that these quasiparticle branches are separated by the (true) energy gap $\left|\Delta_{\mathbf{k}}\right|$. In classical superconductors, $\Delta_{\mathbf{k}}$ implies the off-diagonal-long-range-order (ODLRO) that quantitatively depends on concentration of the BE condensed Cooper pairs. ODLRO is responsible for a dissipationless motion of the charge carriers and simultaneously causes the Meissner effect via the spontaneous gauge symmetry breaking. 


\subsection{The effect of the non-condensed pairs}

In this section we shall study effect of the finite-momentum pairs existing above $T_{\mathrm{c}}$, which no longer develop any ODLRO because there is no BE condensate. Nevertheless, according to (2.4), the single and paired fermions are still mutually dependent. This fact suggests that the previous BCS form (3.5) should be replaced by some corrections originating from the finite momentum pairs. Let us denote the pair propagator by $L(\mathbf{q}, \tau)=-\hat{T}_{\tau}\left\langle\hat{b}(\mathbf{r}, \tau) \hat{b}^{\dagger}(\mathbf{0}, 0)\right\rangle$ and assume its Fourier transform in the following form

$$
L(\mathbf{q}, \omega)=\frac{1}{\omega-E_{\mathbf{q}}-\mathrm{i} \Gamma(\mathbf{q}, \omega)},
$$

where $E_{\mathbf{q}}$ stands for the effective dispersion of pairs and $\Gamma(\mathbf{q}, \omega)$ describes the inverse life-time. Taking into account the equation (2.4), we express the self-energy via the bubble diagram

$$
\Sigma(\mathbf{k}, \omega)=-T \sum_{\mathrm{i} v_{n}, \mathbf{q}} \frac{1}{\omega-\xi_{\mathbf{q}-\mathbf{k}}-\mathrm{i} v_{n}} L\left(\mathbf{q}, \mathrm{i} v_{n}\right),
$$

where $\xi_{\mathbf{q}-\mathbf{k}}=\varepsilon_{\mathbf{q}-\mathbf{k}}-\mu$ and $\mathrm{i} v_{n}$ is the bosonic Matsubara frequency. Since above $T_{\mathrm{c}}$ the preformed pairs are only short-range correlated [21-23], we impose

$$
\left\langle\hat{L}^{\dagger}(\mathbf{r}, t) \hat{L}(\mathbf{0}, 0)\right\rangle \propto \exp \left(-\frac{|t|}{\tau_{\phi}}-\frac{|\mathbf{r}|}{\xi_{\phi}}\right) .
$$

Following T. Senthil and P.A. Lee [22, 23], one can estimate the single particle Green's function $\mathscr{G}(\mathbf{k}, \omega)$ using the following interpolation

$$
\Sigma(\mathbf{k}, \omega)=\Delta^{2} \frac{\omega-\xi_{\mathbf{k}}}{\omega^{2}-\left(\xi_{\mathbf{k}}^{2}+\pi \Gamma^{2}\right)},
$$

where $\Delta$ is the energy gap due to pairing and the other parameter $\Gamma$ is related to damping of the subgap states. In the low energy limit (i.e., for $|\omega| \ll \Delta$ ) the dominant contribution comes from the in-gap quasiparticle whose residue is $Z \equiv\left[1+\Delta^{2} /\left(\pi \Gamma^{2}\right)\right]^{-1}$, whereas at higher energies the BCS-type quasiparticles are recovered. This selfenergy [3.10] can be derived from the microscopic considerations [42] within the two-component model, describing itinerant fermions coupled to the hard-core bosons [43-50].

The other (closely relative) phenomenological ansatz [31, 32]

$$
\Sigma(\mathbf{k}, \omega)=\frac{\Delta^{2}}{\omega+\xi_{\mathbf{k}}+\mathrm{i} \Gamma_{0}}-\mathrm{i} \Gamma_{1}
$$

has been inferred considering the "small fluctuations" regime [26]. Experimental lineshapes of the angle resolved photoemission spectroscopy obtained for the cuprate superconductors at various doping levels and temperatures (including the pseudogap regime) amazingly well coincide with this simple formula 3.11). The gap and the phenomenological parameters $\Gamma_{0}, \Gamma_{1}$ are in general momentum-dependent, but for a given direction in the Brillouin zone one can restrict only to their temperature and doping variations. From now onwards we shall focus on such antinodal region.

In the overdoped samples, $\Gamma_{0}$ can be practically discarded from (3.11) and the remaining parameter $\Gamma_{1}$ simply accounts for $T$-dependent broadening of the Bogoliubov peaks until they disappear just above $T_{\mathrm{c}}$. Physical origin of $\Gamma_{1}$ is hence related to the particle-particle scattering. On the contrary, in the underdoped regime, there exists a pseudogap up to temperatures $T^{*}$ which by far exceed $T_{\mathrm{c}}$. To reproduce the experimental lineshapes, one must then incorporate the other parameter $\Gamma_{0}$ (nonvanishing only above $T_{\mathrm{c}}$ ) which is scaled by $T-T_{\mathrm{c}}$ as shown in figure 1 reproduced from references [31, 32]. Since $\Gamma_{0}$ enters the self-energy (3.11) through the BCS-type structure, its origin is related to the particle-hole scatterings.

We now inspect some consequences of the parametrization (3.11) applicable for the pseudogap regime $T>T_{\mathrm{c}}$ in the underdoped cuprates. Since neither the magnitude of $\Gamma_{1}$ nor $\Delta$ seem to vary over a large temperature region above $T_{\mathrm{c}}$, it is obvious that the qualitative changes are there dominated by scatterings in the particle-hole channel, i.e., due to $\Gamma_{0}$. Roughly speaking, these processes are responsible for filling-in the low energy states upon increasing $T$ as has been evidenced by ARPES [16] and STM [25] 


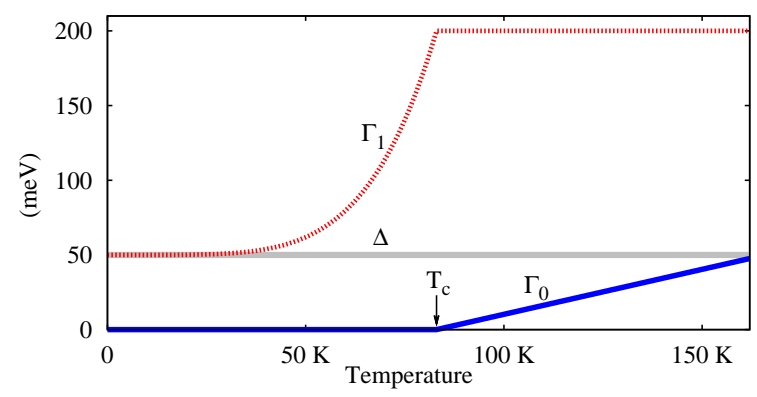

Figure 1. (Color online) Temperature dependence of the phenomenological parameters $\Delta, \Gamma_{0}$ and $\Gamma_{1}$ which, through the self-energy (3.11), reproduce the experimental profiles of the underdoped Bi2212 $\left(T_{\mathrm{C}}=83 \mathrm{~K}\right)$ sample. This fitting is adopted from references [31, 32].

measurements. On a microscopic level, such changes can be assigned to scattering on the preformed pairs.

For analytical considerations, let us rewrite the complex self-energy (3.11) as

$$
\Sigma(\mathbf{k}, \omega)=\left(\omega+\xi_{\mathbf{k}}\right) \frac{\Delta^{2}}{\left(\omega+\xi_{\mathbf{k}}\right)^{2}+\Gamma_{0}^{2}}-\mathrm{i} \Gamma_{\mathbf{k}}(\omega),
$$

where the imaginary part is

$$
\Gamma_{\mathbf{k}}(\omega)=\Gamma_{\mathbf{1}}+\Gamma_{0} \frac{\Delta^{2}}{\left(\omega+\xi_{\mathbf{k}}\right)^{2}+\Gamma_{0}^{2}} .
$$

In what follows we indicate that above $T_{\mathrm{c}}$ the excitation spectrum can consist of altogether three different states, two of them corresponding to the Bogoliubov modes (signifying particle-hole mixing characteristic for the superconducting state) and another one corresponding to the single particle fermion states which form inside the pseudogap. These states start to appear at $T=T_{\mathrm{c}}^{+}$and initially represent heavily overdamped modes containing infinitesimal spectral weight (see reference [22, 23] for a more detailed discussion). Upon increasing temperature, their life-time gradually increases and simultaneously the ingap states absorb more and more spectral weight at the expense of the Bogoliubov quasiparticles. Finally (in the particular case considered here, this happens roughly near $2 T_{\mathrm{c}}$ ) the single particle fermion states become dominant.

Anticipating the relevance of (3.11) to the strongly correlated cuprate materials, one can determine the single particle Green's function $\boldsymbol{G}(\mathbf{k}, \omega)$ and the corresponding spectral function $A(\mathbf{k}, \omega)$. Quasiparticle energies are determined by poles of the Green's function, i.e.,

$$
\omega-\xi_{\mathbf{k}}-\operatorname{Re}\{\Sigma(\mathbf{k}, \omega)\}=0
$$

provided that the imaginary part $\Gamma_{\mathbf{k}}(\omega)$ disappears. We clearly see that the latter requirement cannot be satisfied for $\Gamma_{1} \neq 0$ regardless of $\Gamma_{0}$. Formally this means that the life-time of herein discussed quasiparticles is not infinite. Let us check these eventual (finite life-time) quasiparticle states determined through (3.14). Using the self-energy (3.11), the condition (3.14) is equivalent to

$$
\left(\omega-\xi_{\mathbf{k}}\right)-\left(\omega+\xi_{\mathbf{k}}\right) \frac{\Delta^{2}}{\left(\omega+\xi_{\mathbf{k}}\right)^{2}+\Gamma_{0}^{2}}=0 .
$$

In general, there appear three solutions (figure2) depending on temperature via the parameter $\Gamma_{0}$.

Superconducting region. The fitting procedure [31, 32] has estimated that the parameter $\Gamma_{0}$ vanishes in the superconducting state $T \leqslant T_{\mathrm{c}}$. Under such conditions, 3.15) yields the standard BCS poles at $E_{\mathbf{k}}= \pm \sqrt{\xi_{\mathbf{k}}^{2}+\Delta^{2}}$. Due to $\Gamma_{1} \neq 0$, they show up in the spectral function $A(\mathbf{k}, \omega)$ as Lorentzians whose broadening corresponds to the inverse life-time of the Bogoliubov modes. Owing to $T$-dependence of $\Gamma_{1}$ (see 


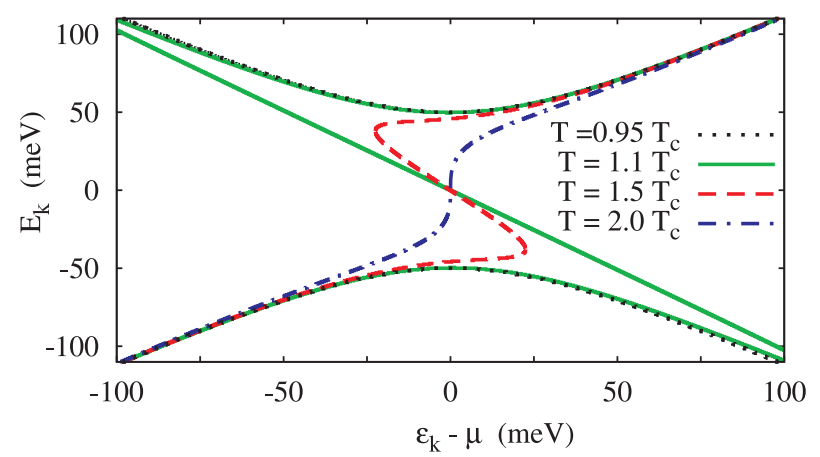

Figure 2. (Color online) Dispersion of $\omega=E_{\mathbf{k}}$ representing the solutions of equation (3.14) for $T / T_{\mathrm{C}}=0.95$ (dotted line), 1.1, 1.5 and 2 (solid curves as described) obtained for the parameters used in references [31, 32]. We notice three different crossings [3.15), two of them related to the Bogoliubov modes and additional one appearing in between.

figure 1), the broadening of these peaks increases upon approaching $T_{\mathrm{c}}$ from below, albeit $A(\mathbf{k}, \omega=0)=0$. Experimentally this process can be observed as the smearing of the coherence peaks [1].

Pseudogap regime. With the appearance of $\Gamma_{0} \neq 0$ above $T_{\mathrm{c}}$, the real part of the self-energy becomes a continuous function of $\omega$ (see figure 3). Consequently, besides the Bogoliubov modes, we now obtain an additional crossing located in-between. Figure 2 shows the representative dispersion curves obtained for $1.1 T_{\mathrm{c}}, 1.5 T_{\mathrm{c}}, 2 T_{\mathrm{c}}$ and compared with the superconducting state (dotted line). We observe either the three branches or just the single one at sufficiently high temperatures when the spectral function $A(\mathbf{k}, \omega)$ evolves to a single peak structure.

As some useful example, let us study the Fermi momentum $\mathbf{k}_{\mathrm{F}}$, when (3.15) simplifies to

$$
\omega\left(1-\frac{\Delta^{2}}{\omega^{2}+\Gamma_{0}^{2}}\right)=0
$$

In this case, we obtain: a) two symmetric quasiparticle energies at $\omega_{ \pm}= \pm \tilde{\Delta}$, where $\tilde{\Delta} \equiv \sqrt{\Delta^{2}-\Gamma_{0}^{2}}$, and b) the in-gap state at $\omega_{0}=0$. The corresponding imaginary parts $\Gamma_{\mathbf{k}}(\omega)$ are

$$
\begin{aligned}
& \Gamma_{\mathbf{k}}\left(\omega_{ \pm}\right)=\Gamma_{1}+\Gamma_{0}, \\
& \Gamma_{\mathbf{k}}\left(\omega_{0}\right)=\Gamma_{1}+\frac{\Delta^{2}}{\Gamma_{0}} .
\end{aligned}
$$

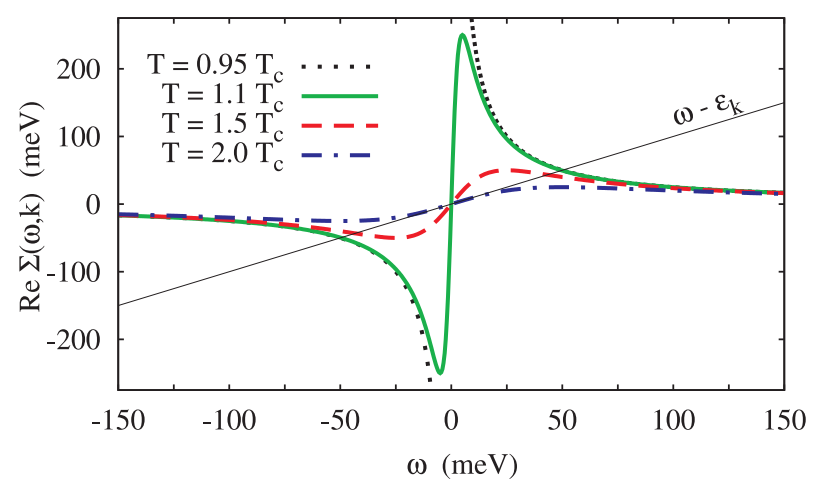

Figure 3. (Color online) The real part of the self-energy $\Sigma(\mathbf{k}, \omega)$ for $\varepsilon_{\mathbf{k}}=\mu$ at several representative temperatures $T / T_{\mathrm{C}}=0.95$ (dotted line) and 1.1, 1.5, 2.0 (as denoted). Below $T_{\mathrm{C}}$ there exist two poles at $\omega= \pm \Delta$ whereas for $T>T_{\mathrm{C}}$, we obtain altogether three crossings which at higher temperature merge into a single one. 


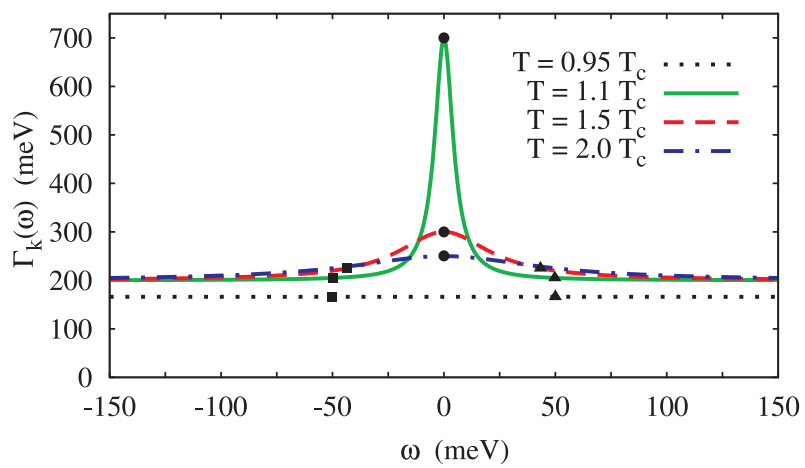

Figure 4. (Color online) The imaginary part $\Gamma_{\mathbf{k}}(\omega)$ for the same set of parameters as used in figure 3 The filled symbols indicate the value of $\Gamma_{\mathbf{k}}(\omega)$ and position of the crossings $\omega=E_{\mathbf{k}}$ of the lower Bogoliubov mode (squares), in-gap state (circles) and the upper Bogoliubov branch (triangles).

Since $\Gamma_{1}$ does not vary above $T_{\mathrm{c}}$, the temperature dependence of $\Gamma_{\mathbf{k}}^{-1}\left(\omega_{i}\right)$ is controlled by $\Gamma_{0}$. Using the experimental estimations [31, 32], we thus find the qualitatively opposite temperature variations of $\Gamma_{\mathbf{k}}\left(\omega_{ \pm}\right)$ and $\Gamma_{\mathbf{k}}\left(\omega_{0}\right)$ shown in figure 5 These quantities correspond to the life-times of quasiparticles and, therefore, we conclude that:

a) in-gap quasiparticles are forbidden for the superconducting state due to vanishing $\Gamma_{\mathbf{k}}^{-1}\left(\omega_{0}\right)=0$ (in other words, spectrum consists of just the Bogoliubov modes typical of the BCS theories),

b) in the pseudogap state above $T_{\mathrm{c}}$, where $\Delta \neq 0$ and $\Gamma_{0} \neq 0$, besides the Bogoliubov branches there emerge in-gap states which initially at $T_{\mathrm{c}}^{+}$represent the heavily overdamped modes.

At a first glance, our conclusions seem to be in conflict with the ARPES data, which have not reported any pronounced in-gap features. Nevertheless, various studies of the pseudogap clearly revealed a rather negligible temperature dependence of $\Delta(T)$ upon passing $T_{\mathrm{c}}$ (at least for the anti-nodal areas). Instead of closing this gap, the low energy states are gradually filled-in [25]. Such a behavior can be thought as an indirect signature of the in-gap states, which for increasing temperatures absorb more and more spectral weight. To support this conjecture, we illustrate in figure 6 an ongoing transfer of the spectral weights between the Bogoliubov quasiparticles and in-gap states. Using (3.11), we show the spectral function $A(\mathbf{k}, \omega)$ subtracting its value at $T_{\mathrm{c}}$ in analogy to the detailed experimental discussion by $\mathrm{T}$. Kondo et al. [16]. In-gap states emerge around $\omega_{0}$ and gradually gain the spectral weight (figure 7) simultaneously increasing their life-time.

Intrinsic broadening of the in-gap states [53] unfortunately obscures their observation by the spectroscopic tools at temperatures close to $T_{\mathrm{c}}$. These states might be, however, probed indirectly. T. Senthil and P.A. Lee [22, 23] suggested that such states could be responsible for the magnetooscillations observed ex-

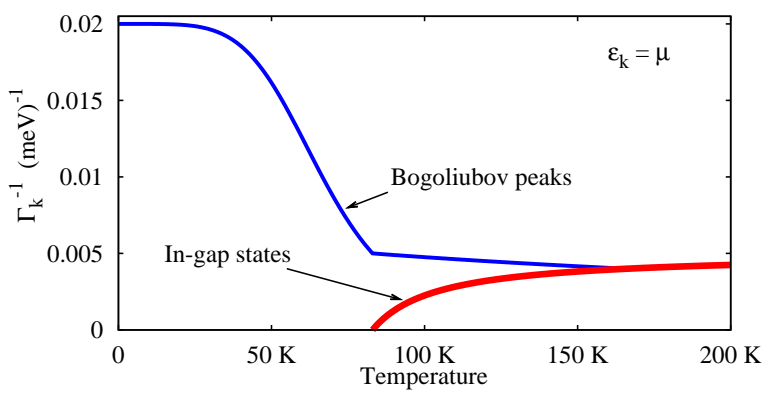

Figure 5. (Color online) Temperature dependence of the inverse broadening $\Gamma_{\mathbf{k}}^{-1}$ which corresponds to the effective life-time of the Bogoliubov modes (thin line) and the in-gap states (thick curve) obtained for $\varepsilon_{\mathbf{k}}=\mu$. 


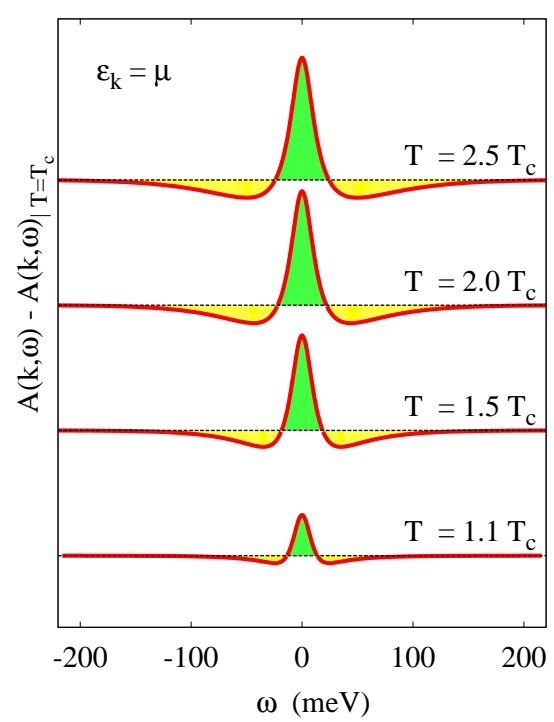

Figure 6. (Color online) Transfer of the spectral weight from the Bogoliubov quasiparticle peaks towards the in-gap states obtained using (3.11) for $\varepsilon_{\mathbf{k}}=\mu$. Temperature dependence of the total transferred spectral weight is shown in figure7

perimentally by N. Doiron-Leyraud et al. [51, 52]. They indicated that pair-coherence extending only over short spatial- and temporal length naturally implies the pair decay (scattering) into the in-gap fermion states. This line of reasoning has been also followed by some other groups [53, 54].

\section{Microscopic toy model}

Pairing of the cuprate superconductors occurs on a local scale, practically between the nearest neighbor lattice sites. To account for an interplay between the paired and unpaired charge carriers taking place in the pseudogap regime we consider here the following simplified picture

$$
\hat{H}_{\mathrm{loc}}=\varepsilon_{0} \sum_{\sigma} \hat{c}_{\sigma}^{\dagger} \hat{c}_{\sigma}+E_{0} \hat{b}^{\dagger} \hat{b}+\left(\Delta \hat{b}^{\dagger} \hat{c}_{\downarrow} \hat{c}_{\uparrow}+\text { h.c. }\right) \text {, }
$$

where $\hat{c}_{\sigma}^{(\dagger)}$ correspond to the unpaired fermions and $\hat{b}^{(\dagger)}$ to the pairs (hard-core bosons). We assume that in the pseudogap state, neither the fermions nor the hard-core boson pairs are long-living because of their mutual scattering by the Andreev charge exchange term. The same type of scattering, although in

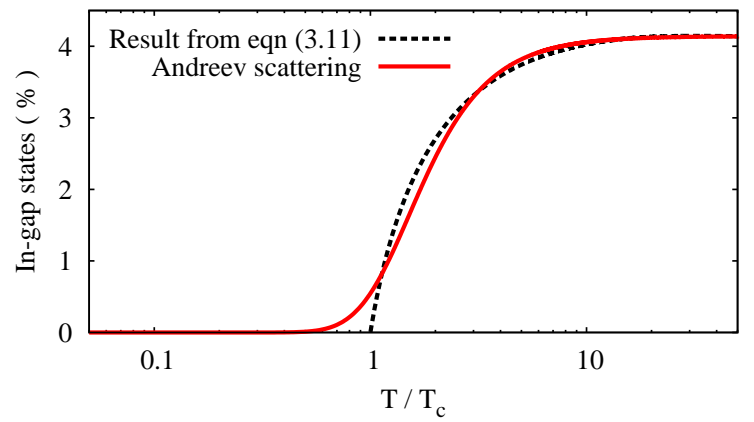

Figure 7. (Color online) Spectral weight corresponding to the in-gap states obtained from the phenomenological ansatz 3.11) for $\varepsilon_{\mathbf{k}}=\mu$ (dotted curve) and solution of the toy model 4.1) for $\varepsilon_{0}=0=E_{0}$ (solid line). 
the momentum space, has been considered in reference [22, 23] within the lowest order diagrammatic treatment. On a microscopic footing, the Hamiltonian (4.1) can be regarded as the effective low energy description of the plaquettized Hubbard model [47, 48].

Neglecting the itinerancy of the charge carriers, we can obtain a rigorous solution for a given local cluster (not to be confused with the individual copper sites in $\mathrm{CuO}_{2}$ planes [50]). Exact diagonalization of the Hilbert space yields the following single particle Green's function [42]

$$
G(\omega)=\frac{\mathcal{Z}_{\mathrm{QP}}}{\omega-\varepsilon_{0}}+\frac{1-\mathfrak{Z}_{\mathrm{QP}}}{\omega-\varepsilon_{0}-\frac{|\Delta|^{2}}{\omega+\varepsilon_{0}-E_{0}}} .
$$

Let us notice that the second term on rhs of (4.2) acquires the same structure as imposed by (3.11). In the present case, no imaginary terms appear but the structure of the Green's function (4.2) mimics the role of $\Gamma_{0}$. Formally, it describes the bonding and antibonding states originating from the Andreev scattering and besides that we also have a remnant of the non-interacting propagator $\left[\omega-\varepsilon_{0}\right]^{-1}$ whose spectral weight is given by $\mathcal{Z}_{\mathrm{QP}}$.

The quasiparticle weight $\mathcal{Z}_{\mathrm{QP}}$ depends on occupancies of the fermion and boson levels. As an example, we explore here the symmetric (i.e., half-filled) case with $\varepsilon_{0}=0$ and $E_{0}=0$ when $Z_{\mathrm{QP}}=2 /[3+$ $\left.\cosh \left(|\Delta| / k_{\mathrm{B}} T\right)\right]$. Assuming the typical ratio $|\Delta| / k_{\mathrm{B}} T_{\mathrm{c}}=4$, we plot in figure 7 the temperature dependence of the unpaired states contribution $\mathcal{Z}_{\mathrm{QP}}$ to the spectrum. We find a very good agreement between our simple treatment and the estimations using the self-energy (3.11). It means that the parameter $\Gamma_{0}$ introduced in references [31, 32] and the local Andreev-type scattering considered here account for the very same particle-hole processes inducing the in-gap states. Transfer of the spectral weight from the paired to unpaired states (figure 7) confirms the qualitative agreement over a broad temperature region and the indication for the same critical point.

For more realistic comparison of the present study with the experimental data [16], one obviously has to consider the itinerant charge carriers. As a natural improvement of the local solution (4.2) we would expect the following type of Green's function

$$
G(\mathbf{k}, \omega)=\frac{\mathcal{Z}_{\mathrm{QP}}(\mathbf{k})}{\omega-\varepsilon_{\mathbf{k}}}+\sum_{\mathbf{q}} \frac{\left[1-\mathcal{Z}_{\mathrm{QP}}(\mathbf{k})\right] f(\mathbf{q}, \mathbf{k})}{\omega-\varepsilon_{\mathbf{k}}-\frac{\left|\Delta_{\mathbf{k}}\right|^{2}}{\omega+\varepsilon_{\mathbf{q}}-\mathbf{k}^{-}-E_{\mathbf{q}}}},
$$

where $T$-dependent coefficients $f(\mathbf{q}, \mathbf{k})$ should be determined via the many-body techniques. Approaching $T_{\mathrm{c}}$ from above the predominant influence comes from $\mathbf{q} \rightarrow \mathbf{0}$ bosons and then we notice that (4.3) reduces to the ansatz (3.11). Such results have been recently reported from the dynamical mean field calculations for the Hubbard model [55, 56].

\section{Conclusions and outlook}

We have shown that the pairing ansatz [3.11, widely used for fitting the experimental ARPES profiles, above $T_{\mathrm{c}}$ corresponds to the pair scattering inducing the single particle fermion states inside the pseudogap. Temperature dependent phenomenological parameter $\Gamma_{0}$ is found to control the transfer of the spectral weight from the Bogoliubov quasiparticles to the unpaired in-gap states. To model such a process on a microscopic level, we have considered the scenario in which the local pairs are scattered into single fermions via the Andreev conversion [22, 23, 47, 48, 50]. We have found a unique relation between the transferred spectral weight (from the paired to unpaired quasiparticles) with the non-bonding state $Z_{\text {QP }}$. It would be instructive to extend the present analysis onto the case of k-dependent energy gap. Such a problem would be closely related to the issue of Fermi arcs, i.e., partially reconstructed pieces of the Fermi surface, and to nontrivial angular dependence of the pseudogap [1].

\section{Acknowledgements}

Author is indebted for the fruitful discussions with Adam Kamiński, Roman Micnas, Julius Ranninger, and Karol I. Wysokiński. This work is supported by the National Science Centre in Poland through the projects DEC-2014/13/B/ST3/04451. 


\section{References}

1. Kaminski A., Kondo T., Takeuchi T., Gu G., Philos. Mag. B, 2014, 95, 453; doi 10.1080/14786435.2014.906758

2. Gaebler J.P., Stewart J.T., Drake T.E., Jin D.S., Perali A., Pieri P., Strinati G.C., Nat. Phys., 2010, 6, 569; doi $10.1038 /$ nphys1709

3. Uemura Y.J., Le L.P., Luke G.M., Sternlieb B.J., Wu W.D., Brewer J.H., Riseman T.M., Seaman C.L., Maple M.B., Ishikawa M., Hinks D.G., Jorgensen J.D., Saito G., Yamochi H., Phys. Rev. Lett., 1991, 66, 2665; doi 10.1103/PhysRevLett.66.2665

4. Orenstein J., Corson J., Oh S., Eckstein J.N., Ann. Phys. (Leipzig), 2006, 15, 596; doi 10.1002/andp.200510202

5. Corson J., Mallozzi R., Orenstein J., Eckstein J.N., Bozovic I., Nature, 1999, 398, 221; doi 10.1038/18402

6. Wang Y., Li L., Ong N.P., Phys. Rev. B, 2006, 73, 024510; doi 10.1103/PhysRevB.73.024510

7. Xu Z.A., Ong N.P., Wang Y., Takeshita T., Uchida S., Nature, 2000, 406, 486; doi 10.1038/35020016

8. Kanigel A., Chatterjee U., Randeria M., Norman M.R., Koren G., Kadowaki K., Campuzano J., Phys. Rev. Lett., 2008, 101, 137002; doi 10.1103/PhysRevLett.101.137002.

9. Shi M., Bendounan A., Razzoli E., Rosenkranz S., Norman M.R., Campuzano J.C., Chang J., Mansson M., Sassa Y., Claesson T., Tjernberg O., Patthey L., Momono N., Oda M., Ido M., Guerrero S., Mudry C., Mesot J., Eur. Phys. Lett., 2009, 88, 27008; doi 10.1209/0295-5075/88/27008

10. Lee J., Fujita K., Schmit A.R., Kim C.K., Eisaki H., Uchida S., Davis J.C., Science, 2009, 325, 1099; doi $10.1126 /$ science.1176369

11. Bergeal N., Lesueur J., Aprili M., Faini G., Contour J.P., Leridon B., Nat. Phys., 2008, 4, 608; doi 10.1038/nphys1017

12. Yuli O., Asulin I., Kalchaim Y., Koren G., Millo O., Phys. Rev. Lett., 2009, 103, 197003; doi 10.1103/PhysRevLett.103.197003

13. Li L., Wang Y., Komiya S., Ono S., Ando Y., Gu G.D., Ong N.P., Phys. Rev. B, 2010, 81, 054510; doi 10.1103/PhysRevB.81.054510

14. Iye T., Nagatochi T., Ikeda R., Matsuda A., J. Phys. Soc. Jpn., 2010, 79, 114711; doi 10.1143/JPSJ.79.114711

15. Bernardi E., Lascilfari A., Ragimonti A., Romano L., Scavini M., Oliva C., Phys. Rev. B, 2010, 81, 064502; doi 10.1103/PhysRevB.81.064502

16. Kondo T., Khasanov R., Takeuchi T., Schmalian J., Kaminski A., Nature, 2009, 457, 296; doi 10.1038/nature07644

17. Dubroka A., Rössle M., Kim K.W., Malik V.K., Munzar D., Basov D.N., Schafgans A.A., Moon S.J., Lin C.T., Haug D., Hinkov V., Keimer B., Wolf Th., Storey J.G., Tallon J.L., Bernhard C., Phys. Rev. Lett., 2011, 106, 047006; doi 10.1103/PhysRevLett.106.047006

18. Kirzhner T., Koren G., Sci. Rep., 2014, 4, 6244; doi 10.1038/srep06244

19. Moon S.J., Lee Y.S., Schafgans A.A., Chubukov A.V., Kasahara S., Shibauchi T., Terashima T., Matsuda Y., Tanatar M.A., Prozorov R., Thaler A., Canfield P.C., Bud'ko S.L., Sefat A.S., Mandrus D., Segawa K., Ando Y., Basov D.N., Phys. Rev. B, 2014, 90, 014503; doi 10.1103/PhysRevB.90.014503

20. Cilento F., Dal Conte S., Coslovich G., Peli S., Nembrini N., Mor S., Banfi F., Ferrini G., Eisaki H., Chan M.K., Dorow C.J., Veit M.J., Greven M., van der Marel D., Comin R., Damascelli A., Rettig L., Bovensiepen U., Capone M., Giannetti C., Parmigiani F., Nat. Commun., 2014, 5, 4353; doi 10.1038/ncomms5353

21. Franz M., Nat. Phys., 2007, 3, 686; doi $10.1038 /$ nphys739

22. Senthil T., Lee P.A., Phys. Rev. Lett., 2009, 103, 076402; doi 10.1103/PhysRevLett.103.076402

23. Senthil T., Lee P.A., Phys. Rev. B, 2009, 79, 245116; doi 10.1103/PhysRevB.79.245116

24. Mamedov T.A., de Llano M., Philos. Mag. B, 2014, 94, 4102; doi 10.1080/14786435.2014.979903

25. Fischer O., Kugler M., Maggio-Aprile I., Berthod Ch., Renner Ch., Rev. Mod. Phys., 2007, 79, 353; doi 10.1103/RevModPhys.79.353

26. Abrahams E., Red M., Woo J.W.F., Phys. Rev. B, 1970, 1, 208; doi 10.1103/PhysRevB.1.208

27. Schmid A., Z. Phys., 1970, 231, 324; doi 10.1007/BF01397514

28. Ranninger J., Robin J.M., Physica C, 1995, 253, 279; doi 10.1016/0921-4534(95)00515-3

29. Emery V.J., Kivelson S.A., Nature, 1995, 374, 434; doi 10.1038/374434a0

30. Tchernyshyov O., Phys. Rev. B, 1997, 56, 3372; doi 10.1103/PhysRevB.56.3372.

31. Norman M.R., Randeria M., Ding H., Campuzano J.C., Phys. Rev. B, 1998, 57, 11093; doi 10.1103/PhysRevB.57.R11093

32. Franz M., Millis A.J., Phys. Rev. B, 1998, 58, 14572; doi 10.1103/PhysRevB.58.14572.

33. Fujimoto S., J. Phys. Soc. Jpn., 2002, 71, 1230; doi 10.1143/JPSJ.71.1230

34. Domański T., Ranninger J., Phys. Rev. Lett., 2003, 91, 255301; doi 10.1103/PhysRevLett.91.255301.

35. Domański T., Ranninger J., Phys. Rev. B, 2001, 63, 134505; doi 10.1103/PhysRevB.63.134505

36. Chubukov A.V., Norman M.R., Millis A.J., Abrahams E., Phys. Rev. B, 2007, 76, 180501; doi 10.1103/PhysRevB.76.180501

37. Chen Q.J., Stajic J., Tan S.N., Levin K., Phys. Rep., 2005, 412, 1; doi 10.1016/j.physrep.2005.02.005 
38. Cichy A., Micnas R., Ann. Phys. (New York), 2014, 347, 207; doi 10.1016/j.aop.2014.04.014.

39. Pizio O., Sokołowski S., Sokołowska Z., J. Chem. Phys., 2014, 140, 174706; doi 10.1063/1.4873438

40. Matsui H., Sato T., Takahashi T., Wang S.-C., Yang H.-B., Ding H., Fujii T., Watanabe T., Matsuda A., Phys. Rev. Lett., 2003, 90, 217002; doi 10.1103/PhysRevLett.90.217002.

41. Chatterjee U., Shi M., Ai D., Zhao J., Kanigel A., Rosenkranz S., Raffy H., Li Z.Z., Kadowaki K., Hinks D.G., Xu Z.J., Wen J.S., Gu G., Lin C.T., Claus H., Norman M.R., Randeria M., Campuzano J., Nat. Phys., 2009, 5, 1456; doi $10.1038 /$ nphys1200

42. Domański T., Phys. Rev. A, 2011, 84, 023634; doi 10.1103/PhysRevA.84.023634

43. Friedberg R., Lee T.D., Phys. Rev. B, 1989, 40, 6740; doi 10.1103/PhysRevB.40.6745

44. Micnas R., Ranninger J., Robaszkiewicz S., Rev. Mod. Phys., 1990, 62, 113; doi 10.1103/RevModPhys.62.113

45. Micnas R., Phys. Rev. B, 2007, 76, 184507; doi 10.1103/PhysRevB.76.184507

46. Geshkenbein V.B., Ioffe L.B., Larkin A.I., Phys. Rev. B, 1997, 55, 3173; doi 10.1103/PhysRevB.55.3173

47. Altman E., Auerbach A., Phys. Rev. B, 2002, 65, 104508; doi 10.1103/PhysRevB.65.104508

48. Mihlin A., Auerbach A., Phys. Rev. B, 2009, 80, 134521; doi 10.1103/PhysRevB.80.134521.

49. Le Hur K., Rice T.M., Ann. Phys. (New York), 2009, 324, 1452; doi 10.1016/j.aop.2009.02.004

50. Ranninger J., Domański T., Phys. Rev. B, 2010, 81, 014514; doi 10.1103/PhysRevB.81.014514

51. Doiron-Leyraud N., Proust C., LeBoeuf D., Levallais J., Bonnemaison J.-B., Liang R., Bonn D.A., Hardy W.N., Taillefer L., Nature, 2007, 447, 565; doi 10.1038/nature05872

52. Taillefer L., J. Phys.: Condens. Matter, 2009, 21, 164212; doi 10.1088/0953-8984/21/16/164212

53. Ranninger J., Romano A., Phys. Rev. B, 2010, 82, 054508; doi 10.1103/PhysRevB.82.054508

54. Micklitz T., Norman M.R., Phys. Rev. B, 2009, 80, 220513; doi 10.1103/PhysRevB.80.220513

55. Imada M., Yamaji Y., Sakai S., Motome Y., Ann. Phys. (Berlin), 2001, 523, 629; doi 10.1002/andp.201100028.

56. Sakai S., Civelli M., Nomura Y., Imada M., Phys. Rev. B, 2015, 92, 180503(R); doi 10.1103/PhysRevB.92.180503

\title{
Стани квазічастинок, керованих розсіюванням на попередньо сформованих електронних парах
}

\author{
Т. Доманьский \\ Інститут фізики, Університет Марії Кюрі-Складовської, 20-031 Люблін, Польща
}

Проаналізовано еволюцію спектру збудження однієї частинки слабо легованих купратних надпровідників поблизу антинодальної зони, беручи до уваги температури вищі і нижчі за фазовий перехід. Досліджено феноменологічну самоенергію, яка відтворює дані ARPES (кутової фотоемісійної спектроскопії). Показано, що при температурах, вищих за критичну, така процедура передбачає перехід спектральної ваги від квазічастинок типу Боголюбова до загасаючих станів всередині щілини. Окрім цього подано певні мікроскопічні аргументи, які пояснюють такий процес.

Ключові слова: флуктуації надпровідності, квазічастинки Боголюбова, псевдощілина 


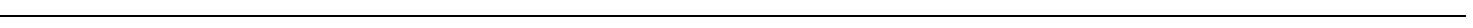

